\title{
DYNAMICS OF HAEMATOPOIETIC STEM CELLS MODEL
}

The article deals with the haematopoietic stem cells model with delay introduced by M. C. Mackey [1]. Our aim is to establish the new conditions under which the equilibria of the model are stable or unstable. The model without delay is also investigated.

Keywords: Haematopoietic stem cells, equilibrium, stability, differential system, delay argument.

\section{Introduction}

The population of haematopoietic stem cells gives rise to all of the differentiated elements of the blood (see [1, 2]): the white blood cells, red blood cells and platelets; which may be either actively proliferating or in a resting phase. After entering the proliferating phase, a cell is committed to undergo cell division at a fixed time $\tau$ later. The generation time $\tau$ is assumed to consist of four phases: the pre-synthesis phase, the DNA synthesis phase, the post-synthesis phase and the mitotic phase. Just after the division, both daughter cells go into the resting phase. Once in this phase, they can either return to the proliferating phase and complete the cycle or die before ending the cycle. The dynamics of the model is governed by the nonlinear differential system with delay argument:

$$
\begin{aligned}
& \frac{d N}{d t}=-\delta N(t)-\beta(N(t)) N(t)+ \\
& +2 e^{-\gamma \tau} \beta(N(t-\tau)) N(t-\tau), \\
& \frac{d P}{d t}=-\gamma P(t)-\beta(N(t)) N(t)- \\
& -e^{-\gamma \tau} \beta(N(t-\tau)) N(t-\tau), t \geq 0,
\end{aligned}
$$

where $\beta(N)$ is a monotone decreasing function and has the explicit form of a Hill function:

$$
\beta(N)=\beta_{0} \frac{\theta^{n}}{\theta^{n}+N^{n}}, n=1,2, \ldots .
$$

The symbols in (1) have a following interpretation: $N$ is the number of cells in nonproliferative phase, $P$ is the number of cycling proliferating cells, $\gamma$ is the rate of cells loss from proliferative phase, $\delta$ is the rate of cells loss from nonproliferative phase, $\tau$ is the time spent in the proliferative phase, $\beta$ is the feedback function which represents the rate of recruitment from nonproliferative phase, $\beta_{0}$ is the maximum recruitment rate and $\theta, n$ control the shape of the feedback function. We assume that $\delta, \gamma, \tau, \beta_{0}, \theta \in(0, \infty)$.

\section{Equilibria of System (1) Without Delay}

For $\tau=0$ the system (1) has a form

$$
\begin{aligned}
& \frac{d N}{d t}=-\delta N(t)-\beta(N(t)) N(t), \\
& \frac{d P}{d t}=-\gamma P(t), t \geq 0 .
\end{aligned}
$$

The equilibrium points of (2) we obtain from the system

$$
\begin{aligned}
& -\delta N+\beta(N) N=0, \\
& -\gamma P=0 .
\end{aligned}
$$

The system (2) has the trivial equilibrium $E_{0}=(N, P)=(0,0)$ and the nontrivial equilibrium $E=\left(N^{*}, P^{*}\right)=\left(\beta^{-1}(\delta), 0\right)$.

The value $N^{*}=\beta^{-1}(\delta)$ we can obtain from the equation

$$
\begin{aligned}
& \beta\left(N^{*}\right)=\beta_{0} \frac{\theta^{n}}{\theta^{n}+N^{* n},} \\
& \beta\left(\beta^{-1}(\delta)\right)=\beta_{0} \frac{\theta^{n}}{\theta^{n}+N^{* n}}, \\
& \delta\left(\theta^{n}+N^{* n}\right)=\beta_{0} \theta^{n}, \\
& N^{*}=\theta\left(\frac{\beta_{0}}{\delta}-1\right)^{\frac{1}{n}}, \beta_{0}>\delta .
\end{aligned}
$$

\footnotetext{
* Julia Stoberova

Department of Crisis Management, Faculty of Special Engineering, University of Zilina, Slovakia, E-mail: julia.stoberova@gmail.com
} 


\section{Stability of Equilibria Without Dealy}

Theorem 1. [2] Assume that $\delta \in\left(0, \beta_{0}\right)$. Then the system (2) has the trivial equilibrium $E_{0}=(0,0)$ which is unstable and a positive one $E=\left(N^{*}, 0\right)$ which is asymptotically stable.

Theorem 2. Suppose that $\delta \in\left(\beta_{0}, \infty\right)$. Then the system (2) has the trivial equilibrium $E_{0}=(0,0)$ which is asymptotically stable.

Proof. The matrix of the linearized system (2) has a form

$$
A=\left(\begin{array}{cc}
-\delta+\beta^{\prime}(N) N+\beta(N) & 0 \\
0 & -\gamma
\end{array}\right)
$$

and the matrix of the linearized system around $E_{0}=(0,0)$ is given by

$$
A_{1}=\left(\begin{array}{cc}
-\delta+\beta^{\prime}(0) 0+\beta(0) & 0 \\
0 & -\gamma
\end{array}\right)=\left(\begin{array}{cc}
-\delta+\beta_{0} & 0 \\
0 & -\gamma
\end{array}\right) \text {. }
$$

Then the characteristic equation of the linearized system of (2) around $E_{0}$ is

$$
\begin{aligned}
& \operatorname{det}\left(A_{1}-\lambda I\right)=0, \\
& \left|\begin{array}{cc}
-\delta+\beta_{0}-\lambda & 0 \\
0 & -\gamma-\lambda
\end{array}\right|=0, \\
& (\lambda+\gamma)\left(\lambda-\beta_{0}+\delta\right)=0 .
\end{aligned}
$$

This equation has two roots given by

$\lambda_{1}=-\gamma<0, \lambda_{2}=\beta_{0}-\delta<0,\left(\delta>\beta_{0}\right)$.

Thus the point $E_{0}=(0,0)$ is asymptotically stable equilibrium of the system (2).

\section{Model with Delay $\tau>0$}

Using

$$
\delta(N)=\beta(N) N
$$

we rewrite system (1) as follows

$$
\begin{aligned}
& \frac{d N}{d t}=-\delta N(t)-\alpha(N(t))+2 e^{-\gamma \tau} \alpha(N(t-\tau)), \\
& \frac{d P}{d t}=-\gamma P(t)+\alpha(N(t))-e^{-\gamma \tau} \alpha(N(t-\tau)), t \geq 0 .
\end{aligned}
$$

\section{We set}

$$
s=\frac{t}{\tau} \quad \text { i.e } t=s \tau
$$

and we get

$$
\begin{aligned}
& N(t)=N(s \tau)=u(s), N(t-\tau)=N(\tau(s-1))=u(s-1), \\
& P(t)=P(s \tau)=v(s), \\
& \frac{d N(t)}{d t}=\frac{d u(s)}{d t}=\frac{d u(s)}{d s} \frac{d s}{d t}=\dot{u}(s) \frac{1}{\tau}, \\
& \frac{d P(t)}{d t}=\frac{d v(s)}{d t}=\frac{d v(s)}{d s} \frac{d s}{d t}=\dot{v}(s) \frac{1}{\tau} .
\end{aligned}
$$

Using the relations above the system (3) is transformed into the next one

$$
\begin{aligned}
& \dot{u}(t)=\tau\left[-\delta u(t)-\alpha(u(t))+2 e^{-\gamma \tau} \alpha(u(t-1))\right], \\
& \dot{v}(t)=\tau\left[-\gamma v(t)+\alpha(u(t))-e^{-\gamma \tau} \alpha(u(t-1))\right], \mathrm{t} \geq 0 .
\end{aligned}
$$

In the next consideration we will use the root $\tau$ of the equation

$$
\beta_{0}\left(2 e^{-\gamma \tau}-1\right)-\delta=0
$$

We get

$$
\begin{gathered}
e^{-\gamma \tau}=\frac{1+\frac{\delta}{\beta_{0}}}{2}, \\
-\gamma \tau=\ln \frac{1+\frac{\delta}{\beta_{0}}}{2}, \\
\tau=\frac{1}{\gamma} \ln \frac{2}{1+\frac{\delta}{\beta_{0}}} .
\end{gathered}
$$

We will use the notation

$$
\bar{\tau}=\frac{1}{\gamma} \ln \frac{2}{1+\frac{\delta}{\beta_{0}}}
$$

Lemma 1. Suppose that $0<\delta<\beta_{0}$. Then $\bar{\tau}>0$.

Proof. We have

$\frac{\delta}{\beta_{0}}<1,1+\frac{\delta}{\beta_{0}}<2$ and $\frac{2}{1+\frac{\delta}{\beta_{0}}}>1$ implies that $\bar{\tau}>0$.

Lemma 2. Assume that $0<\delta<\beta_{0}$. Then for $0<\tau<\bar{\tau}$ it holds that

$\beta_{0}\left(2 e^{-\gamma \tau}-1\right)-\delta>0$.

Proof. Since $\tau<\bar{\tau}$, then 


$$
\begin{aligned}
& \gamma \tau<\ln \frac{2}{1+\frac{\delta}{\beta_{0}}}, \\
& 2 e^{-\gamma \tau}>1+\frac{\delta}{\beta_{0}}, \\
& \beta_{0}\left(2 e^{-\gamma \tau}-1\right)-\delta>0 .
\end{aligned}
$$

Lemma 3. Suppose that $0<\delta<\beta_{0}$. Then for $0<\tau<\bar{\tau}$ it holds that $2 e^{-\gamma \tau}-1>0$.

Proof. Since $\beta_{0}\left(2 e^{-\gamma \tau}-1\right)-\delta>0$. and $\beta_{0}>0, \delta>0$, it follows that

$$
2 e^{-\gamma \tau}-1>0
$$

Lemma 4. Assume that $0<\delta<\beta_{0}$. Then for $\tau>\bar{\tau}$ the next inequality holds

$\beta_{0}\left(2 e^{-\gamma \tau}-1\right)-\delta<0$.

Proof. Since $\tau>\bar{\tau}$, then we get

$$
\begin{gathered}
\gamma \tau>\ln \frac{2}{1+\frac{\delta}{\beta_{0}}}, \\
2 e^{-\gamma \tau}<1+\frac{\delta}{\beta_{0}}, \\
\beta_{0}\left(2 e^{-\gamma \tau}-1\right)-\delta<0 .
\end{gathered}
$$

\section{Equilibria of System (4)}

If we take $\alpha(u)=\beta(u) u$, then the equilibria of (4) we obtain from the system

$$
\begin{aligned}
& -\delta u-\beta(u) u+2 e^{-\gamma \tau} \beta(u) u=0, \\
& -\gamma v-\beta(u) u-e^{-\gamma \tau} \beta(u) u=0 .
\end{aligned}
$$

The first equation of (5) is satisfied for

$u=0$ or $-\delta-\beta(u)+2 e^{-\gamma \tau} \beta(u)=0$.

For $u=0$ from the second equation of (5) we get that $v=0$. So the system (4) has the trivial equilibrium $(u, v)=(0,0)$.

The positive equilibrium of (4) we obtain from the system

$-\delta-\beta(u)+2 e^{-\gamma \tau} \beta(u)=0$,

$-\gamma v+\beta(u) u-e^{-\gamma \tau} \beta(u) u=0$.
From the first equation it follows that

$$
\beta(u)=\frac{\delta}{2 e^{-\gamma \tau}-1}
$$

According to Hill function we have

$$
\begin{aligned}
& \beta_{0} \frac{\theta^{n}}{\theta^{n}+u^{n}}=\frac{\delta}{2 e^{-\gamma \tau}-1}, \\
& u^{n}=\beta_{0} \theta^{n} \frac{2 e^{-\gamma \tau}-1}{\delta}-\theta^{n}, \\
& u=\theta\left(\frac{\beta_{0}\left(2 e^{-\gamma \tau}-1\right)-\delta}{\delta}\right)^{\frac{1}{n}} .
\end{aligned}
$$

From the second equation we get

$$
\begin{aligned}
& v=\frac{u}{\gamma}\left(\beta(u)-e^{-\gamma \tau} \beta(u)\right)=\frac{u}{\gamma} \beta(u)\left(1-e^{-\gamma \tau}\right)= \\
& =\frac{d u}{\gamma}\left(\frac{1-e^{-\gamma \tau}}{2 e^{-\gamma \tau}-1}\right)=\frac{\delta \theta}{\gamma}\left(\frac{\beta_{0}\left(2 e^{-\gamma \tau}-1\right)-\delta}{\delta}\right)^{\frac{1}{n}} . \\
& \cdot \frac{1-e^{-\gamma \tau}}{2 e^{-\gamma \tau}-1} .
\end{aligned}
$$

Put $u=u^{*}, v=v^{*}$. For $0<\delta<\beta_{0}, 0<\tau<\bar{\tau}$ the point $E^{*}=\left(u^{*}, v^{*}\right)$ is a positive equilibrium of the system (4).

\section{Trivial Equilibrium of System (4)}

The linearized system of (4) around the trivial equilibrium $(0,0)$ is given by

$$
\begin{aligned}
& \dot{u}(t)=\tau\left[\delta-\alpha^{\prime}(0) u(t)+2 e^{-\gamma \tau} \alpha^{\prime}(0) u(t-1)\right], \\
& \dot{v}(t)=\tau\left[-\gamma v(t)+\alpha^{\prime}(0) u(t)-e^{-\gamma \tau} \alpha^{\prime}(0) u(t-1)\right] .
\end{aligned}
$$

The characteristic equation of the linearized system of (4) is

$$
\begin{aligned}
& \left|\begin{array}{cc}
\tau\left[-\delta-\alpha^{\prime}(0)+2 e^{-\gamma \tau} \alpha^{\prime}(0)\right]-\lambda & 0 \\
\tau\left[\alpha^{\prime}(0)-e^{-\gamma \tau} \alpha^{\prime}(0)\right] & -\tau \gamma-\lambda
\end{array}\right|=0, \\
& (\lambda+\tau \gamma)\left(\lambda+\tau\left[\delta+\alpha^{\prime}(0)-2 e^{-\gamma \tau} \alpha^{\prime}(0)\right]=0 .\right.
\end{aligned}
$$

The roots of the equation above are

$\lambda_{1}=-\tau \gamma, \lambda_{2}=\tau\left[-\delta-\alpha^{\prime}(0)+2 e^{-\gamma \tau} \alpha^{\prime}(0)\right]$.

Because $\alpha^{\prime}(0)=\beta(0)=\beta_{0}$, we have

$$
\lambda_{1}=-\tau \gamma, \lambda_{2}=\tau\left[-\delta+\beta_{0}\left(2 e^{-\gamma \tau}-1\right)\right] .
$$

Theorem 3. [2] Suppose $0<\delta<\beta_{0}$. Then for $0<\tau<\bar{\tau}$ the trivial equilibrium $(0,0)$ of the system $(4)$ is unstable. 
Proof. Since $\lambda_{1}=-\tau \gamma<0$ and with regard to Lemma 2 we have that $\lambda_{2}=\tau\left[-\delta+\beta_{0}\left(2 e^{-\gamma \tau}-1\right)\right]>0$ then equilibrium $(0,0)$ is unstable.

Theorem 4. Suppose that $0<\delta<\beta_{0}$. Then for $\tau>\bar{\tau}$ the trivial equilibrium $(0,0)$ of the system (4) is asymptotically stable.

Proof. We have $\lambda_{1}=-\tau \gamma<0$ and according to Lemma 4 $\lambda_{2}=\tau\left[-\delta+\beta_{0}\left(2 e^{-\gamma \tau}-1\right)\right]<0$. Then the equilibrium $(0,0)$ is asymptotically stable.

\section{Positive Equilibrium of System (4)}

The linearized system of (4) around the positive equilibrium $E^{*}=\left(u^{*}, v^{*}\right)$ is given by

$$
\begin{aligned}
& \dot{u}(t)=\tau\left[\delta-\alpha^{\prime}\left(u^{*}\right) u(t)+2 e^{-\gamma \tau} \alpha^{\prime}\left(u^{*}\right) u(t-1)\right], \\
& \dot{v}(t)=\tau\left[-\gamma v(t)+\alpha^{\prime}\left(u^{*}\right) u(t)-e^{-\gamma \tau} \alpha^{\prime}\left(u^{*}\right) u(t-1)\right] .
\end{aligned}
$$

The characteristic equation of the linearized system of (4) around $E^{*}$ is

$$
\begin{aligned}
& \left|\begin{array}{cc}
\tau\left[-\delta-\alpha^{\prime}\left(u^{*}\right)+2 e^{-\gamma \tau} e^{-\lambda} \alpha^{\prime}(u *)\right]-\lambda & 0 \\
\tau\left[\alpha^{\prime}\left(u^{*}\right)-e^{-\gamma \tau} e^{-\lambda} \alpha^{\prime}\left(u^{*}\right)\right] & -\tau \gamma-\lambda
\end{array}\right|=0, \\
& (\lambda+\tau \gamma)\left(\lambda+\tau\left[\delta+\alpha^{\prime}\left(u^{*}\right)-2 e^{-\gamma \tau} e^{-\lambda} \alpha^{\prime}\left(u^{*}\right)\right]=0 .\right.
\end{aligned}
$$

From this equation we obtain

$$
\lambda_{1}=-\tau \gamma \text { or } \lambda+\tau\left[\delta+\left(1-2 e^{-\gamma \tau} e^{-\lambda}\right) \alpha^{\prime}\left(u^{*}\right)\right]=0 .
$$

We will be interested in real part of the roots of equation

$$
\lambda+\tau \delta+\tau\left[\left(1-2 e^{-\gamma \tau} e^{-\lambda}\right) a^{\prime}\left(u^{*}\right)\right]=0 .
$$

Theorem 5. Suppose that $\alpha^{\prime}\left(u^{*}\right)<0$ and $-\alpha^{\prime}\left(u^{*}\right)(1+$ $\left.+2 e^{-\gamma \tau}\right)<\delta<\beta_{0}$ for $0<\tau<\bar{\tau}$. Then the equilibrium $E^{*}=\left(u^{*}\right.$, $\left.v^{*}\right)$ is asymptotically stable.

Proof. Let $\lambda=\mu+i v$ be the root of (6). Then we have

$$
\begin{aligned}
& \mu+i v+\tau \delta+\tau\left(1-2 e^{-\gamma \tau} e^{-(\mu+i v)}\right) \alpha^{\prime}\left(u^{*}\right)=0, \\
& \mu+\tau \delta+\tau \alpha^{\prime}\left(u^{*}\right)-2 \tau e^{-\gamma \tau} \alpha^{\prime}\left(u^{*}\right) e^{-\mu} \cos v+ \\
& +i\left(v+2 \tau e^{-\gamma \tau} \alpha^{\prime}\left(u^{*}\right) e^{-\mu} \sin v\right)=0 .
\end{aligned}
$$

Separating the real and imaginary parts leads to

$$
\begin{aligned}
& \mu+\tau \delta+\tau \alpha^{\prime}\left(u^{*}\right)-2 \tau e^{-\gamma \tau} \alpha^{\prime}\left(u^{*}\right) e^{-\mu} \cos v=0, \\
& v+2 \tau e^{-\gamma \tau} \alpha^{\prime}\left(u^{*}\right) e^{-\mu} \sin v=0 .
\end{aligned}
$$

We assume that (7) is satisfied for some $\mu \geq 0$. Then we get

$$
\tau \delta+\tau \alpha^{\prime}\left(u^{*}\right)-2 \tau e^{-\gamma \tau-\mu} \alpha^{\prime}\left(u^{*}\right) \cos v \leq 0
$$

and

$$
2 \tau e^{-\gamma \tau-\mu} \alpha^{\prime}\left(u^{*}\right) \cos v \geq \delta+\alpha^{\prime}\left(u^{*}\right) .
$$

Since

$$
\delta>-a^{\prime}\left(u^{*}\right)\left(1+2 e^{-\gamma \tau}\right)>-a^{\prime}\left(u^{*}\right),
$$

then $\delta+\alpha^{\prime}\left(u^{*}\right)>0$. From (8) we have

$$
\cos \nu \leq \frac{\delta+\alpha^{\prime}\left(u^{*}\right)}{2 e^{-\gamma \tau-\mu} \alpha^{\prime}\left(u^{*}\right)}<0
$$

Because $-1 \leq \cos v \leq 1$, we obtain

$$
\begin{aligned}
& -1 \leq \frac{\delta+\alpha^{\prime}\left(u^{*}\right)}{2 e^{-\gamma \tau-\mu} \alpha^{\prime}\left(u^{*}\right)}<0, \\
& -2 e^{-\gamma \tau-\mu} \alpha^{\prime}\left(u^{*}\right) \geq \delta+\alpha^{\prime}\left(u^{*}\right)>0, \\
& \delta \leq-\alpha^{\prime}\left(u^{*}\right)\left(1+2 e^{-\gamma \tau-\mu}\right) .
\end{aligned}
$$

Since $1+2 e^{-\gamma \tau-\mu} \leq 1+2 e^{-\gamma \tau}$, we get

$\delta \leq-\alpha^{\prime}\left(u^{*}\right)\left(1+2 e^{-\gamma \tau}\right)$.

This is a contradiction with condition $\delta>-a^{\prime}\left(u^{*}\right)\left(1+2 e^{-\gamma \tau}\right)$ of the theorem. So it cannot be $\mu \geq 0$. Thus $\lambda=\mu+i v$ has a negative real part. From it follows that $E^{*}$ is asymptotically stable.

Theorem 6. Suppose that $\alpha^{\prime}\left(u^{*}\right) \geq 0$ and $\alpha^{\prime}\left(u^{*}\right)\left(2 e^{-\gamma \tau}-\right.$ $-1)<\delta<\beta_{0}$ for $0<\tau<\bar{\tau}$. Then the equilibrium $E^{*}=\left(u^{*}, v^{*}\right)$ is asymptotically stable.

Proof. Assume that (7) is satisfied for some $\mu \geq 0$. If $\alpha^{\prime}\left(u^{*}\right)=$ $=0$, them from (8) we obtain that $\delta \leq 0$, which is a contradiction with condition that $\delta>0$. So we will assume that $\alpha^{\prime}\left(u^{*}\right)>0$. From (8) we get

$$
\cos \nu \geq \frac{\delta+\alpha^{\prime}\left(u^{*}\right)}{2 e^{-\gamma \tau-\mu} \alpha^{\prime}\left(u^{*}\right)}>0 .
$$

Since $|\cos v| \leq 1$, we have

$$
\begin{aligned}
& \frac{\delta+\alpha^{\prime}\left(u^{*}\right)}{2 e^{-\gamma \tau-\mu} \alpha^{\prime}\left(u^{*}\right)} \leq 1, \\
& \delta+\alpha^{\prime}\left(u^{*}\right) \leq 2 e^{-\gamma \tau-\mu} \alpha^{\prime}\left(u^{*}\right), \\
& \delta \leq \alpha^{\prime}\left(u^{*}\right)\left(2 e^{-\gamma \tau-\mu}-1\right) .
\end{aligned}
$$


Because

$$
2 e^{-\gamma \tau-\mu}-1 \leq 2 e^{-\gamma \tau}-1
$$

we obtain that

$$
\delta \leq a^{\prime}\left(u^{*}\right)\left(2 e^{-\gamma \tau}-1\right)
$$

which is a contradiction with condition that

$$
\delta>a^{\prime}\left(u^{*}\right)\left(2 e^{-\gamma \tau}-1\right)
$$

So it cannot be $\mu \geq 0$. Thus $\mu<0$ and $E^{*}$ is asymptotically stable equilibrium.

\section{Simulation}

For $\beta_{0}=2, \delta=1, \gamma=1, \tau=1, \theta=1, n=2$ the system (4) is given by

$$
\begin{aligned}
& \dot{u}(t)=-u(t)-\frac{2 u(t)}{1+u^{2}(t)}+\frac{4}{e} \frac{u(t-1)}{1+u^{2}(t-1)}, \\
& \dot{v}(t)=-v(t)-\frac{2 u(t)}{1+u^{2}(t)}+\frac{2}{e} \frac{u(t-1)}{1+u^{2}(t-1)}, t \geq 0 .
\end{aligned}
$$

Fig. 1 shows three solutions of (9) in the phase plane which converge to asymptotically stable equilibrium $E=(0,0)$, (Theorem $4)$.

For $n=2, \theta=1, \beta_{0}=2, \delta=1, \gamma=1, \tau=0.1$ the system (4) has a form

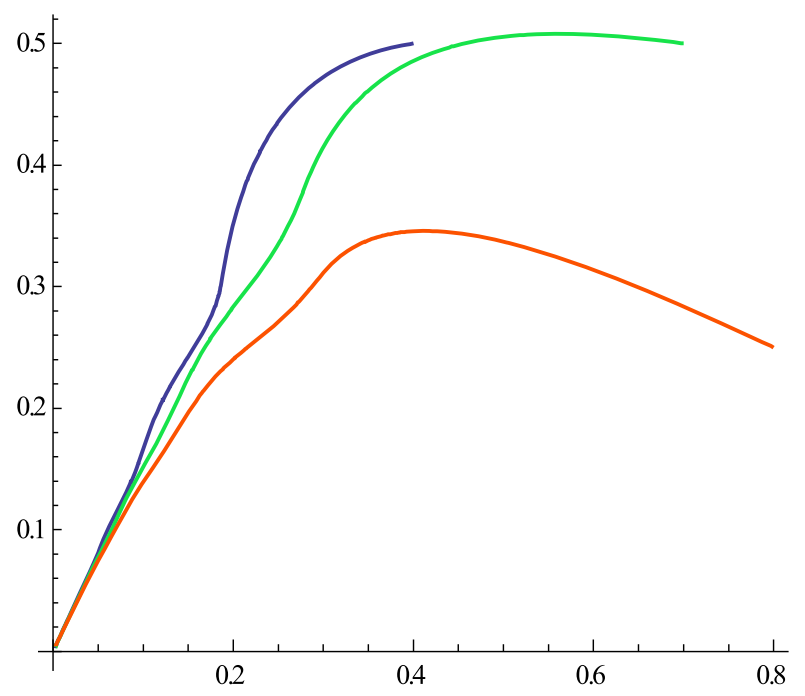

Fig. 1 Solutions of (9) in the phase plane $\dot{u}(t)=0.1\left[-u(t)-\frac{2 u(t)}{1+u^{2}(t)}+4 e^{-0.1} \frac{u(t-1)}{1+u^{2}(t-1)}\right]$,

$\dot{v}(t)=0.1\left[-v(t)-\frac{2 u(t)}{1+u^{2}(t)}-2 e^{-0.1} \frac{u(t-1)}{1+u^{2}(t-1)}\right]$,

$t \geq 0$.

Fig. 2 shows three solutions of (10) in the phase plane which converge to asymptotically stable equilibrium $E^{*}=(u, v)=(0.7870$, 0.0925), (Theorem 6).

\section{Conclusion}

Since the differentiation and interaction in a population of haematopoietic stem cells is very complex and complicated, it is difficult to comprehend the large scale dynamics of this process without the formal structure of a mathematical model.

The main results of the article are new, extend and improve some results in [2]. For example the conditions of Theorem 6 are satisfied for $n=2, \theta=1, \beta_{0}=2, \delta=1, \gamma=1, \tau=0.1$ or for $n=2, \theta=1, \beta_{0}=2, \delta=1.5, \gamma=1, \tau=0.05$ or for $n=2, \theta=1$, $\beta_{0}=2, \delta=1.2, \gamma=1, \tau=0.1$ But these parameters do not satisfy condition of Theorem 3.1 in [2].

\section{Acknowledgement}

The research was supported by the Grant 1/1260/12 of the Scientific Grant Agency of the Ministry of Education of the Slovak Republic.

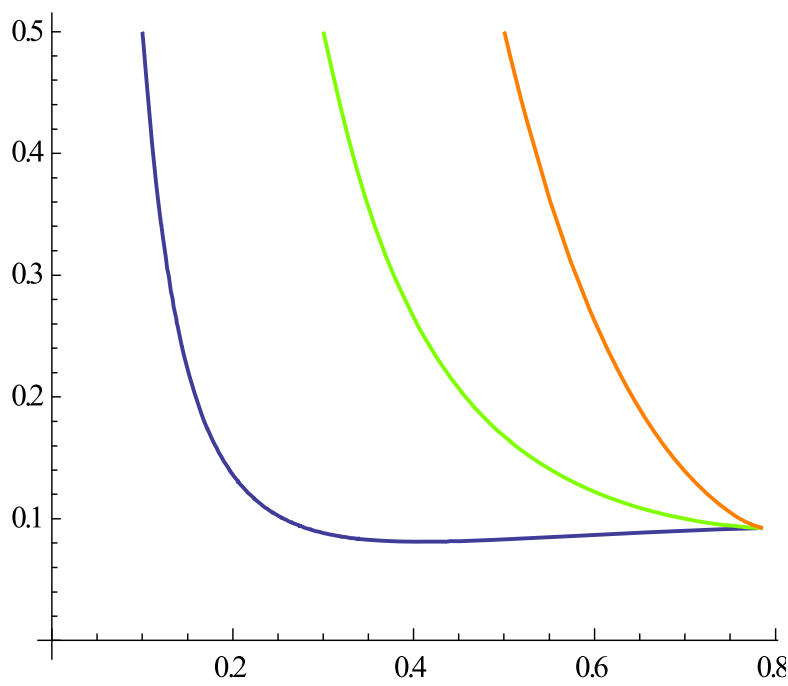

Fig. 2 Solutions of (10) in the phase plane 


\section{References}

[1] MACKEY, M. C.: Unified Hypothesis for the Origin Aplastic Anemia and the Periodic Hematopoietics, Blood 51, 5 (1978).

[2] TALIBI ALAOUI, H., YAFIA, R.: Stability and Hopf Bifurcation in an Approachable Haematopoietic Stem Cells Model, Mathematical Biosciences. 206 (2007), pp. 176-184.

[3] DOROCIAKOVA, B., OLACH, R.: Existence of Positive Solutions of Delay Differential Equations, Tatra Mt. Math. Publ. 43, 2009, pp. 63-70.

[4] LEVIN, S. A., HALlAM, T. G., GROSS, L. J.: Applied Mathematical Ecology, Springer - Verlag, Berlin : New York : London : Tokyo, 1989.

[5] MARUSIAK, P., OLACH, R.: Functional Differential Equations (in Slovak). EDIS - University of Zilina, 2000.

[6] OLACH, R.: Observation of a Feedback Mechanism in a Population Model, Nonlinear Analysis, 41 (2000), pp. 539-544. 